\title{
Justicia, eficiencia y comunidades campesinas
}

Enrique Delgado Flores

\section{Introducción}

Diego García-Sayán ${ }^{1}$, narra la historia de un juez de paz, ante quien se presenta la cuestión de determinar la propiedad de una vaca, que era discutida por dos vecinos. El juez de la historia, con mucha sabiduría decide dejar libre al animal, que a paso lento pero seguro, se dirige al corral de uno de los litigantes, dueño sin duda del animal.

La moraleja de la historia, estriba en la aceptación de la capacidad para resolver conflictos por parte de operadores no-profesionales del derecho. Como podrá apreciarse el final es feliz, y la solución eficiente, pues nuestro juez cumplió con el fin último del proceso 'la paz social', disipó la incertidumbre asignando una titularidad de un modo más eficiente (si es posible) que la del mercado, y a pesar del tiempo que tardó la vaca en dirigirse a su corral, este debe ser sin duda alguna uno de los procesos de mayor celeridad.

El problema que planteamos en el presente trabajo, no es ciertamente novedoso, habida cuenta de la facilidad con la que cualquier persona -ligada o no al quehacer judicial- puede constatar el estado real de nuestro sistema de administración de justicia, y muy especialmente el referente al Poder Judicial ${ }^{2}$.

1 García-Sayán, 1987, pp. 39-43.

2 Según una encuesta de Apoyo S.A., el $92 \%$ de los peruanos tiene desconfianza de los magistrados del Poder Judicial. Citado por Bustamante, 1993, p. 19. 
Existe una amplia literatura sobre el tema, la cual ha estudiado el problema, centrándose sobre todo en el Poder Judicial. Así, existen estudios respecto a la percepción del ciudadano frente a este órgano, otros que hablan de la corrupción, algunos de la falta de celeridad procesal; etc. Existen también otros trabajos que, preferentemente desde una perspectiva sociológica, analizan el tema de los mecanismos de administración de justicia "populares" y "alternativos" ${ }^{2}$

Dentro del segundo grupo de estudios mencionados, hay quienes sostienen que una forma de mejorar el funcionamiento de la administración de justicia en nuestro país es la "desjudicialización" de una serie de procesos, pues la reducción de los conflictos "justiciables" en el Poder Judicial, permitirá solucionar algunos de sus problemas, sobre todo los derivados de la excesiva carga procesal ${ }^{4}$.

En la misma línea, hace poco tiempo, el Congreso aprobó una ley que permite que muchos de los denominados "procesos no contenciosos" sean resueltos por notarios públicos; y por otro lado la Constitución Política establece la existencia de una "jurisdicción arbitral", como medio de promoción de mecanismos alternativos de solución de conflictos, y reconoce a las autoridades de las comunidades campesinas y nativas ciertas potestades jurisdiccionales, en un intento de comprensión del carácter pluricultural de nuestro país.

Por otro lado, desde noviembre de 1995 existe dentro del mismo Poder Judicial una Secretaría Ejecutiva, que aunque legalmente está subordinada a la Comisión Ejecutiva del Poder Judicial (integrada sobre todo por magistrados), ha encarnado en la práctica amplísimos poderes, y viene trabajando en un intento de reforma "estructural" del Poder Judicial.

Para nosotros es claro que el problema de la administración de justicia en nuestro país, es mucho más amplio que el ámbito del Poder Judicial o dicho en otros términos, no creemos que baste la 'modernización' de este poder del Estado para mejorar el sistema de administración de justicia. También se hace necesario involucrar en este proceso

3 Al hablar de mecanismos de solución de conflictos populares se habla sobre todo de comunidades campesinas y nativas, rondas comunales y urbanas. Cuando se habla de mecanismos alternativos se refiere principalmente a la conciliación, mediación y arbitraje.

4 Por ejemplo en el estudio del Instituto de Economía de Libre Mercado realizado por Alberto Bustamante. Justicia Alternativa. 1993. 
a todas las instituciones que realizan labores conexas (Ministerio Público, INPE, Policía Nacional, entre otros), y "alternativas" (cortes arbitrales, organismos de promoción de la conciliación como las DEMUNAS, comunidades campesinas, etc. $)^{5}$

En este trabajo, empezamos, por realizar un análisis de la eficiencia como atributo de la administración de justicia, para lo cual utilizamos algunas herramientas de la economía, y luego contrastamos los criterios allí expuestos con uno de los mecanismos "alternativos", la justicia en las comunidades campesinas.

Debemos reconocer que, de modo consciente, hemos obviado muchas de las carencias más notorias del Poder Judicial (especialmente el vinculado a la apreciación general de "corrupción") y nos hemos centrado sobre todo en el problema del exceso de demanda, como causa de algunos de los costes de la administración de justicia.

En lo referente a la segunda parte del trabajo, debemos reconocer que nuestra ignorancia en los métodos de las ciencias sociales, nos ha impedido realizar un análisis más rico de los problemas vinculados a la pluralidad cultural y jurídica.

\section{La eficiencia en la administración de justicia}

La economía peruana, como señala el artículo $58^{\circ}$ de la Constitución Política, es "social de mercado", lo que significa que su desarrollo está librado principalmente a la "actividad privada" dentro de un marco de libertad en la iniciativa privada.

Aunque la libertad, entendida en su más amplia acepción como la posibilidad de "buscar nuestro propio bien a nuestra propia manera, mientras no intentemos privar de sus bienes a otros o frenar sus esfuerzos para obtenerla" ${ }^{\prime}$, constituye el marco referencial básico para la existencia de la actividad privada que reporta beneficios a los privados (y

5 Así por ejemplo, José Dellepiane, Secretario Ejecutivo de la Comisión Ejecutiva del Poder Judicial, señalaba, que el ciudadano común achacaba las carencias de órganos como el INPE o la Policía Judicial al Poder Judicial, pues lo entiende como parte de un todo. En ciclo de conferencias internacionales sobre la reforma de la administración de justicia. Hotel Las Américas, Lima, agosto 1996.

6 Stuart Mill, 1980, p. 33. 
por tanto a la colectividad) ${ }^{7}$, es necesaria además la existencia de ciertas "reglas de juego", que actúen como incentivos y garantías de la actividad privada, North denomina a estas reglas "instituciones"8.

Según el mismo North, el desarrollo económico puede ser explicado en virtud al desarrollo institucional de las naciones ${ }^{9}$. Estas instituciones funcionan básicamente dando "forma a la interacción humana. Por consiguiente estructuran incentivos en el actuar humano, sea político, social o económico" ${ }^{10}$. Según el mismo autor, las instituciones pueden ser formales o informales ${ }^{11}$. Dentro de la primera categoría se encontrarían las reglas políticas (y judiciales), reglas económicas y los contratos $^{12}$; y en la segunda algunas limitaciones (contraints), como los códigos de conducta, normas de comportamiento y convenciones ${ }^{13}$.

Lo interesante es que ambos tipos de limitaciones pretenden enmarcar el ámbito, dentro del cual el individuo puede diseñar su conducta libremente, y por ende su violación deberá ser pasible de algún tipo de sanción, ya que el "tipo y la eficacia de los mecanismos de exigibilidad determinan la índole total del juego"14.

En nuestro país, desde hace algunos años, se viene realizando un esfuerzo por construir un marco institucional adecuado al tipo de desarrollo económico que se pretende, mediante el incentivo de la actividad privada; es por ello que se ha citado una considerable cantidad de dispositivos que pretenden regular (o en algunos casos "desregular") muchos campos de actividad.

Como debe resultar más o menos claro, no basta con el dictado de las normas, sino, que se requiere que éstas sean cumplidas, es por ello

7 "Un individuo que "intenta solamente su propio beneficio" es conducido por una mano invisible a alcanzar un fin que no formaba parte de sus intenciones. Ni el hecho de que ese fin no formará parte de sus intenciones es siempre malo para la sociedad. Al perseguir sus propios intereses, el individuo promueve a menudo los de la sociedad de un modo más efectivo que cuando intenta directamente promoverlos..." Adam, Smith. The wealth of nations. 1776. Citado por Friedman, 1983, p. 16.

8 North, 1983, p. 16.

9 Ibid., pp. 6-9.

10 Ibidem., p. 3: “... are the humanly devised constraints that shape human interaction. In consequence they structure incentives in human exchange, wheter political, social, or economics".

11 Ibidem., p. 4

12 Ibidem., p. 47.

13 Ibid., p. 36.

14 Ibid., p. 4 " ... the type and effectiveness of enforcement shape the hwole character of the game...". 
que el derecho prevé, una serie de mecanismos coercitivos y represivos que refuercen su cumplimiento. Estos mecanismos, que también son llamados law enforcement, están en manos principalmente de agentes estatales, ya sea, pertenecientes a la administración o al Poder Judicial, $y$ en algunos casos se estipula que las partes en conflicto por incumplimiento de alguna estipulación legal (o cuando exista duda respecto del sentido de una norma), puedan recurrir a otros medios como el arbitraje, por ejemplo.

Resulta lógico que quienes participan como agentes económicos, pretendan conocer las reglas de juego para acceder participar en él; pero no basta el conocimiento, pues se requiere además poseer la certeza o creencia de que dichas reglas van a ser respetadas por todos. Un caso típico es el de la extensión del crédito bancario. En un medio donde no existe confianza en los sistemas legales previstos para la exigibilidad del pago de deudas, los bancos, solo están dispuestos a prestar a las personas con las cuales los une algún vínculo de confianza, de este modo se "oligarquiza la economía" con el consecuente perjuicio para el desarrollo. ${ }^{15}$

Dentro de una economía donde las transacciones operan con un carácter cooperativo, valores como el conservar el buen nombre, pueden funcionar con mayor eficacia que las más perfectas formas de exigibilidad ideadas, este es un caso de funcionamiento de instituciones informales como los códigos de conducta ${ }^{16}$. Sin embargo, dentro de una economía que aspira al desarrollo, las transacciones son cada vez más impersonales, de allí la importancia de contar, tanto con un marco institucional informal que estimule comportamientos eficientes como códigos de conducta, como con un bien desarrollado marco formal, respaldado por un sólido sistema de exigibilidad.

\subsection{El postulado de eficiencia}

El término eficiencia, para los objetivos del presente trabajo, puede ser definido como "la relación entre los beneficios totales de una situación

15 Ejemplo tomado de Trazegnies, 1995, pp. 41-42.

16 Para un análisis de las implicancias del problema de la cooperación, y 'dilema del prisionero’ en un juego repetitivo, véase: North, 1990, pp. 13 y ss. 
y los costes totales de la misma"17. En ese sentido, un organismo público (o de cualquier otro tipo) será eficiente en tanto alcance sus beneficios con el menor costo posible, que incluye no solo los gastos propios con origen en el Presupuesto de la República, sino todos aquellos que realizan los usuarios del servicio.

Debemos aclarar que la situación óptima en eficiencia que puede ser alcanzada no es la situación utópica de un sistema de administración sin injusticias, que no origina costos innecesarios, o donde las sentencias brillen por su excepcional versación jurídica; sino aquella situación más eficiente que no pueda ser mejorada con los recursos existentes sin perjudicar otro bien; en ese sentido será óptima aquella situación en la que no es posible una mejor calidad de las decisiones judiciales, sin incurrir un costo que exceda los recursos disponibles, o alternativamente aquella situación en que no es posible abaratar los costos sin perjudicar la calidad de las decisiones ${ }^{18}$.

El objetivo del Poder Judicial puede ser definido principalmente como la solución de conflictos que surjan en la sociedad (este objetivo será el mismo en cualquier órgano de resolución de conflictos), por lo cual podemos señalar, como Santos Pastor, que lo que hace es brindar un servicio que puede ser denominado "tutela judicial de los derechos"19, que lo brinda en aras de aumentar el "bienestar social".

\subsubsection{El aporte del análisis económico del derecho}

Dentro de los estudios del análisis económico del derecho, el cumplimiento o exigibilidad de las leyes, ha sido un tema recurrente.

Una gran parte de estos estudios, principalmente los realizados por Calabresi ${ }^{20}$, se encuadran dentro del estudio de la responsabilidad civil, como mecanismo simulador de una solución de mercado, cuando

17 Polinsky, 1983, p. 19. Preferimos empezar con esta definición más cercana al sentido común que al uso técnico del término por los economistas, debido a que, emplear el concepto de acuerdo a la idea de "optimalidad paretiana", involucraría introducir el análisis los beneficios privados netos en relación a los beneficios sociales netos, escapando así del área privativa de este análisis inicial, comprendido por el órgano administrador de justicia.

18 Cfr. Pastor, 1993 , p. 42.

19 Pastor, 1993, p. 40.

20 Autor de numerosos estudios sobre el tema, del cual es sin duda una autoridad. Entre sus escritos más populares: Tragic Choices (1978), The Cost of Accidents (1970), etc. 
el trato directo entre las partes interesadas acarrea altos costos. Podemos ver así un primer acercamiento, que caracteriza al Poder Judicial (o al órgano que resuelve un conflicto proveniente de daños), como reasignador de bienes escasos, en especiales situaciones en las cuales existan altos costos de transacción que impidan a las partes negociar directamente $^{21}$.

Dentro de esta perspectiva del análisis económico, ha sido tradicional evaluar, lo costoso que puede significar implementar un law enforcement, considerando las características de los individuos ${ }^{22}$.

Las conclusiones principales de estos estudios, pueden en gran medida resumirse en lo expuesto por Torres López: "(...) no es suficiente que los procedimientos judiciales se limiten a resolver conflictos sino que ello debe hacerse conforme a los criterios y normas que regulan dichas instituciones (...) conforme al postulado de eficiencia" ${ }^{23}$.

Para lograr lo expuesto anteriormente, se requiere que el "uso de un sistema de exigibilidad, no ocasione mayores pérdidas que las derivadas del conflicto mismo"24.

Es importante destacar que las conclusiones acá analizadas provienen principalmente de la denominada corriente "eficientista", que se preocupa sobre todo de alcanzar una mayor eficiencia económica, negando al Derecho la capacidad de manifestarse sobre la justicia. En contra están las críticas de Calabresi, que sostiene la posibilidad de derivar algunas nociones del Derecho a partir del análisis económico ${ }^{25}$.

Uno de los temas de mayor preocupación dentro de este tipo de análisis es el derivado de los costes que originarían los errores judiciales. En ese sentido, se afirma, que su prevención tiene como límite el momento en que se iguala con los costos administrativos que significaría su total erradicación ${ }^{26}$. Creemos que esta afirmación puede ser válida en la corrección de nuestro sistema, aplicada incluso a otros aspectos. Así por ejemplo, se deberá tolerar la supervivencia de un pequeño margen de corrupción, cuando los costes de eliminarla totalmente sean superiores a los que se originen en esta falla.

21 Coase, 1960 , pp. 16 y ss.

22 Ver p.e. Polinsky (1983), capítulo 10, pp. 90-101. y Santos Pastor, 1984, pp. 171172.

23 Torres López, 1987, p. 67.

24 Ibid.

25 Calabresi, 1992 (1984), pp. 90 y ss.

26 Torres López, 1987, p. 68. 


\subsubsection{Concepto amplio de bienestar social}

Como señalamos anteriormente (ver 1.1.), la misión de los órganos de administración de justicia, a partir de la tutela judicial efectiva de derechos, es finalmente acrecentar el bienestar social.

El paradigma neoclásico, subyacente a los estudios de análisis económico, supone a los individuos como maximizadores de beneficios, que al realizar sus elecciones en un contexto de escasez de recursos, preferirán las soluciones más cercanas a su función de utilidad, comprendida tanto por componentes monetarios y no monetarios.

Dentro de este contexto, surge la cuestión referente al conflicto entre la eficiencia (el tamaño de la torta) y la equidad (la forma en que ésta ha de ser distribuida) ${ }^{27}$. Un enfoque netamente eficientista podrá asumir que lo importante, es acrecentar su tamaño, en la idea de que si "el pastel es mayor, todos podrán obtener un trozo más grande" 28 . Sin embargo, como sabemos, los criterios adoptados por el legislador al asignar titularidades, muchas veces incluyen criterios redistributivos y otras razones como la justicia ${ }^{29}$.

Es dentro de este contexto que resulta crucial entender cuál debe ser el criterio que guiaría las soluciones que plantee el derecho, a partir de la intervención de la administración de justicia. Rawls ${ }^{30}$, define su Teoría de la Justicia sobre dos principios:

a) El derecho de todos a una extensa e igual gama de libertades; $y$,

b) El arreglo de las situaciones de desigualdad económica y social, a través de un sistema de mayor beneficio para los menos aventajados, basado en el sistema de ahorros justos, ligados a igualdad de acceso a posiciones y funciones para todos.

En base al segundo principio, resultaría coherente, tanto con una concepción de justicia cercana a equidad como con los objetivos de eficiencia, la existencia de desigualdades, siempre que existan expectativas de mejoría para los menos aventajados. Visto así, resulta claro,

27 Polinsky, 1983, p. 19.

28 Ibid.

29 Calabresi, 1992 (1972), pp. 101 a 108.

30 Rawls, 1979 (1971), pp. 67-68. 
que la justicia es un valor distinto de la eficiencia, que puede ser introducido normativamente.

Torres López, señala, que el problema consiste en dilucidar:

“... si la ciencia económica se constituye sobre la base de asegurar la virtualidad de unos comportamientos que se presumen lo más adecuados con arreglo con el fin supremo de eficiencia o sobre la base de proporcionar las claves que permitan orientar e influir sobre las conductas de manera que se alcancen, por el camino más expedito en cada momento, los diversos objetivos que a su vez han sido discrecionalmente fijados por los sujetos económicos, aun cuando de su propia consecución no se siga necesariamente el equilibrio perfecto del mercado en las condiciones preestablecidas de competencia perfecta"31

Para el presente análisis nosotros reconocemos que el concepto de bienestar social tiene un contenido amplio (e impreciso), pero asumimos que, en todo caso la labor de la administración de justicia es ampliar el bienestar social (o reducir el malestar), mediante la tutela de los derechos de los individuos que han sido atacados ${ }^{32}$.

Respecto del problema entre eficiencia y equidad, reconocemos la existencia de una normatividad que, en supuestos específicos, puede privilegiar objetivos redistributivos o basados en la justicia aun cuando no sean eficientes, siempre y cuando no exista contradicción con el modelo de sociedad adoptado, que privilegia la acción eficiente de los individuos, mediante la protección de la libertad de la actividad privada.

\subsection{La administración de justicia en el Perú}

Como anteriormente definimos la administración de justicia en los términos de un servicio (ver 1.1), para realizar un primer acercamiento, que evalúe sus costes de prestación, analizaremos las funciones de la oferta y demanda.

De manera preliminar señalaremos que uno de los más graves problemas de nuestro Poder Judicial es el exceso de demanda, que a su vez

31 Torres López, 1987, p. 37.

32 Esta concepción es idéntica a la asumida por Pastor Prieto, 1993, p. 40. 
se agrava por las características de la oferta y del servicio ofrecido, que estudiamos a continuación.

\subsubsection{La oferta}

La oferta de este servicio en nuestro país está principalmente en manos del Poder Judicial, aunque como es sabido, existen también otros "ofertantes" de servicios que en un sentido lato podrían calzar en la definición de servicios sustitutos.

Creemos innecesario abundar en las características de la oferta del Poder Judicial que, como se señala, es generalmente ineficiente, pues sus costos administrativos son demasiado elevados. Se indica por ejemplo, que el diseño de los procesos es inadecuado pues sería el origen de problemas tales como la falta de celeridad procesal.

Además se dice que genera a las partes una serie muy grande de costos. Así por ejemplo, Javier de Belaunde señala:

"La justicia no solo es lenta sino incalificable, muchas veces no hace justicia.

Hay problemas de previsibilidad, falta de jurisprudencia orientadora, desubicación del juez ante nuevas áreas del derecho y de la vida social. De otro lado, preocupa una creciente irregularidad en el sistema judicial" 33 .

\subsubsection{Variaciones en la oferta}

Una acción de política pública, destinada a variar la oferta del servicio de administración de justicia, puede enfocar el problema desde dos perspectivas diferentes, que desarrollamos a continuación.

En primer término, puede centrarse en mejorar la calidad del servicio ofrecido realizando acciones distintas, tales como aumentar el número de dependencias, capacitar a los magistrados, proveer de mayores ingresos al Poder Judicial; etc. Estas soluciones suelen ser muy costosas, y aunque posiblemente mejoren el nivel de paz social, es también muy posible que aumenten considerablemente la carga procesal.

33 De Belaunde, 1991, p. 33. 
Otra forma de encarar el problema, que podría resultar más barata, es la consistente en la variación de la oferta de este servicio creando, por decirlo así, una "competencia" entre distintos ofertantes del servicio.

\subsection{Los mecanismos alternativos de solución de conflictos}

En este apartado, haremos una breve reflexión en torno al papel, que pueden jugar los mecanismos de solución de conflictos alternativos más conocidos: la mediación, el arbitraje y la conciliación.

Suponiendo que los individuos tienen un comportamiento racional, que los lleva a elegir las alternativas menos costosas (más beneficiosas), es lógico suponer que el problema de exceso de demanda de los servicios del Poder Judicial puede ser resuelto diversificando la oferta, mediante la promoción de vías alternas de solución que resulten más beneficiosas para los individuos.

Para realizar este examen obviaremos la falta de información de los individuos (desconocimiento de la situación real y manejo por parte de abogados, ver infra. p. 20), y supondremos que todo individuo frente a un conflicto que desea resolver conoce todas las alternativas y mecanismos de solución.

Dada la condición inicial antes expuesta, es lógico que para poder aumentar su demanda, estos servicios alternativos deben ser menos costosos, es decir deberán ofrecer entre, otras cosas, la posibilidad de una solución más rápida y con menor riesgo de error.

En opinión de Bustamante, estos mecanismos suelen ser ventajosos respecto del proceso judicial, en tanto son más expeditivos, y por las características de sus procesos (generalmente no pre-establecido legalmente, ni preclusivo) permiten a las partes una mejor exposición de sus pretensiones y pruebas ${ }^{34}$. Nosotros podríamos agregar, además, que la existencia de un "facilitador" o "juzgador" (o su inexistencia) elegido por las partes reduce las posibilidades de corrupción.

Pero también se puede afirmar que en casos determinados como "en materia de responsabilidad derivada de los accidentes", los procedimientos de autocomposición son "inferiores" -crean menos estigma negati-

34 Bustamante, 1993, pp. 75-78. 
vo- a los procedimiento judiciales ${ }^{35}$. Se trata pues de una situación en la que los medios alternativos, no obstante ser más baratos no ayudan a fortalecer el marco institucional.

Respecto a la presencia de un criterio de eficiencia en las soluciones que se alcancen por medio de estos mecanismos, creemos que debe estar privilegiada, tanto si la solución se basa en la aplicación de normas positivas o esté arreglada a "conciencia". Esto puede deducirse fácilmente, si la solución alcanzada es idéntica a la que hubiese proporcionado el mercado ante la posibilidad de negociar sin costos de transacción entre las partes en disputa.

\subsection{Desjudicialización de procesos}

Una solución a los problemas de exceso de demanda en el Poder Judicial, es la sustracción de competencia de ciertas materias a este poder del Estado.

Recientemente se aprobó un dispositivo que permite la resolución de asuntos no contenciosos por los notarios. Esto, de acuerdo a la posición de la Dra. Lourdes Flores Nano, obedece, a que la «... jurisdicción (que) se ejerce cuando no existe conflicto (sic) (...) no es propiamente tal"36.

El problema, en todo caso, se agudiza en torno a las materias que si incluyen la existencia de un conflicto, en cuyo caso se discute si la desjudicialización debe ser compulsiva, o reservada a la elección de las partes $^{37}$.

En nuestra opinión, la posibilidad de desjudicializar procesos solo debe tener los siguientes límites: en primer lugar, pleitos referidos a bienes cuya titularidad está protegida por una regla de inalienabilidad ${ }^{38}$ (v.gr. la vida, la libertad), vale decir aquellos que no pueden ser objeto de negociación por los individuos; y en segundo lugar cuando, como

35 Pastor, 1993, p. 40.

36 Flores Nano, 1995, p. 266, citando a Alzamora Valdez.

37 Para una confrontación de opiniones véase Bustamante, 1993, pp. 101 y ss.

38 Calabresi y Melamed, diferencian entre reglas de Propiedad, Responsabilidad e Inalienabilidad; estas últimas se presentan cuando "la ley, no solo decide quién es titular de algo y que precio deberá pagarse por ello si es violado o destruido, sino que además regula su venta; por ejemplo estableciendo pre-condiciones para que la venta sea válida o prohibiendo del todo su venta". Calabresi y Melamed, 1992 (1972), p. 76. 
se vio anteriormente, la solución judicial es más efectiva en aras de fortalecer el marco institucional (ver nota 30 ).

\subsection{Otras alternativas}

Dentro de la concepción del sujeto racional que se comporta eficientemente, y considerando que la justicia es un servicio "en el mercado", creemos que una posibilidad adicional de reducción de la demanda de tutela es la posibilidad de hacerla más costosa en ciertos casos.

Esta propuesta solo sería aplicable en el caso de controversias patrimoniales, que impondría a las partes el pago de elevadas sumas de dinero como "derechos", cuando sus pretensiones tengan gran relevancia económica. En nuestra opinión esto no importa un recorte del derecho a una tutela judicial efectiva, ya que deja abierta la posibilidad de alternativas menos costosas y que incluso, como el caso del arbitraje, son denominadas "jurisdicción".

\subsubsection{El comportamiento de la demanda}

Para analizar el comportamiento de la demanda en nuestro país, asumiremos que los usuarios son sujetos racionales, en el sentido usual del término, que buscan maximizar sus beneficios. En este sentido, conociendo de la existencia de servicios sustitutos, sería lógico presumir que la demanda se contrae, por una marcada preferencia a esos otros servicios. Sin embargo, paradójicamente, uno de los grandes problemas del Poder Judicial es precisamente el exceso de demanda del servicio.

$\mathrm{Al}$ respecto se han expuesto diversas explicaciones. Algunos señalan nuestra idiosincrasia inclinada a "litigar". Así, con cierta frecuencia suele citarse que los indígenas, desde épocas muy cercanas a la conquista e inicios de la colonia, litigaban con tanta frecuencia que las actividades conexas se convirtieron en actividad rentable.

Otros estudios, como el de Pásara, sugieren explicaciones originales, como la de presumir, que:

“... ir al Poder Judicial, en muchos casos tiene una útil función amenazante para quien no puede obtener su derecho por 
otra vía; aunque el procedimiento tarde, nadie acepta tranquilamente ser enjuiciado por lo imprevisible que es el resultado" ${ }^{39}$.

Así, el autor citado señala como ejemplo el caso del inquilino, que ante el vencimiento de su contrato demanda al propietario para dilatar el tiempo antes de ser desalojado ${ }^{40}$. Este fenómeno, denominado "pleitos artificiales" 41 , ha sido ampliamente estudiado. Según Santos Pastor son una manifestación de carácter estratégico y se sustancian, a pesar de carecer de base alguna, con la idea de obtener algún tipo de acuerdo ventajoso antes del pleito ${ }^{42}$; la solución estribaría en un desincentivo específico en forma de penalización ${ }^{43}$.

Otra explicación, que da Bustamante, refiere al carácter del servicio sustitutorio ofrecido por los medios alternativos de resolución de conflictos, señalando especialmente causas como la creencia de que es necesario tener mucho dinero para acudir a ellas, o el papel de los abogados en la elección de la vía de solución ${ }^{44}$.

Intentar dar una respuesta del porqué litiga la gente, para poder variar la función de demanda, con respuestas distintas a las ya esbozadas respecto de una variación de la oferta, resulta difícil. Santos Pastor, en su trabajo ya citado, elabora una teoría al respecto, de donde extraemos sus principales conclusiones:

Si no existen costes de litigación, ni de llegar a un acuerdo, y no existe comportamiento estratégico, y las partes son neutrales al riesgo, entonces, solo habrá litigio, si lo ofrecido por el ofensor es menor a la menor cuantía de pretensión por la que el demandado negociaría ${ }^{45}$.

- En una situación similar a la anterior, pero con costes de litigación para ambas partes, habrá acuerdo, si lo ofrecido por el ofensor menos sus costes de litigar, es menor que la menor suma por la que convendría más sus costes de litigar ${ }^{46}$.

39 Pásara, 1982, p. 34.

40 Ibid.

41 Pastor, 1993, p. 143.

42 Ibid, p. 145.

43 Ibid.

44 Bustamante, 1993, p. 76.

45 Pastor, 1993, p. 121.

46 Ibid. p. 123. 
- Si la situación es similar a la anterior, pero existen costos para llegar a un acuerdo, y no para litigar, entonces habrá acuerdo si la cantidad ofrecida por el ofensor menos sus costes por llegar a un acuerdo, es igual o menor que la menor cantidad esperada por el demandante para convenir más sus costos de llegar al acuerdo. ${ }^{47}$

- Si ambas partes tienen aversión al riesgo, las probabilidades de llegar a un acuerdo aumentan ${ }^{48}$.

\section{La justicia en las comunidades campesinas}

Hasta concluir el anterior capítulo, consideramos la existencia de "jurisdicciones", o medios de solución de conflictos alternativos al Poder Judicial, como algo normal e incluso deseable por sus caracteres de eficiencia.

Sin embargo, la existencia de estas vías de solución de conflictos, supone una trasgresión del modelo de Estado basado en la división de tres poderes, mediante la cual se confiere de manera más o menos precisa un cierto tipo de función a cada uno de ellos. Nuestro texto constitucional, establece la "unidad y exclusividad de la función jurisdiccional", y solo reconoce dos jurisdicciones independientes, la militar y la arbitral. Tan fuerte es esta concepción, que en otro lugar se establece la prohibición a cualquier autoridad de avocarse a causas pendientes ante el órgano jurisdiccional.

Sin embargo, al final del capítulo referido al Poder Judicial, el mismo cuerpo de leyes, establece, la potestad de las autoridades de las comunidades campesinas y nativas, para que con el apoyo de las rondas campesinas, puedan ejercer las funciones jurisdiccionales dentro su ámbito territorial de conformidad con el derecho consuetudinario, y sin violar los derechos fundamentales de la persona.

\section{1 ¿Por qué se rompe la unidad de la función jurisdiccional?}

La existencia de una autoridad única, con capacidad de administrar justicia, es una característica de los Estados modernos, y suele señalarse, 
que es un logro de la Revolución Liberal, que alcanza la igualdad sustancial y formal de los individuos ante la ley, mediante la unicidad de la función de administrar justicia, eliminándose la existencia odiosa de fueros privativos como un derecho de casta. Este logro suele vincularse además con la monopolización por parte del Estado de la posibilidad de dictar normas jurídicas.

Como De Trazegnies nos recuerda, la existencia de un Poder Judicial centralizado y único es una necesidad de todo Estado, con prescindencia del modelo que acepte, ya sea uno centralmente planificado, o ya sea que se trate de un Estado Liberal. Respecto de este último, es especialmente interesante recordar la supuesta necesidad de esta unicidad pues:

"No cabe duda que si cada grupo particular puede instaurar su propio orden normativo, no se darán las condiciones de generalización y predictibilidad necesarias para lograr el tejido de intercambios que "arman" la sociedad liberal" 4 "

De acuerdo a un cierto modelo de Estado, una de sus principales funciones, es intercambiar un cierto grupo de servicios, denominados protección y justicia a cambio de renta ${ }^{50}$.

Esta potestad, se la irroga el Estado en base a un monopolio de la violencia, que le permite, establecer las titularidades, y también por medio del uso de la "justicia" (o más precisamente la administración de justicia), intervenir en la economía ${ }^{51}$, ya sea ratificando las asignaciones iniciales o variándolas.

Sin embargo, debemos recordar, que el Estado se encuentra limitado por el coste-oportunidad de sus gobernados, pues siempre existirán rivales potenciales para ofrecer la misma clase de servicios ${ }^{52}$.

En los casos observados en el anterior capítulo, estos rivales del Estado no están representados por otros Estados extranjeros, sino por particulares al interior del mismo. La posibilidad de que los particulares, prefieran a estos otros viene determinada por los mayores beneficios que le signifiquen.

49 Trazegnies, 1977 , p. 80.

50 North, 1981, p. 38.

51 Coase, 1960, p. 24.

52 North, 1981, p. 39. 


\subsection{Pluralidad cultural}

La decisión de dotar a grupos particulares dentro de un estado de potestades jurisdiccionales, no obedece únicamente a razones de eficiencia, en muchos casos como el de las comunidades campesinas y nativas, obedece más bien a un reconocimiento de la denominada pluralidad cultural, aunque ello no niega que también puedan resultar decisiones eficientes.

En este trabajo, no discutiremos los problemas referentes a la pluralidad cultural, sino que lo asumiremos como un dato proporcionado por la realidad y verificable.

La sola existencia de órdenes culturales distintos, muchas veces constituye un conflicto, que se acrecienta cuando se pretende superponer un mismo orden jurídico a varios grupos con patrones culturales distintos, máxime si lo que se pretende es imponer los criterios de uno de los subgrupos al resto. El derecho (de la sociedad que sea) emite juicios de valor sobre las costumbres, calificándolos como buenos o malos ${ }^{53}$, la calificación de un hecho por el derecho de otra cultura, implica realizar una nueva calificación de estos hechos, la cual puede ser contradictoria con la calificación primigenia realizada.

Pero, además, el derecho es portador de ciertos valores, que suelen preconizarse como de valor universal, así por ejemplo se habla de los derechos fundamentales de las personas como derechos "naturales del hombre"; y del mismo modo el derecho del grupo preponderante, que desea ser el derecho oficial de un Estado, puede contener valoraciones, que no necesariamente serán compartidas por todos los otros grupos, lo cual como es lógico puede ser también fuente de conflictos.

\subsection{Análisis del artículo $149^{\circ}$ de la Constitución Política del Perú}

La norma en análisis, señala la existencia de una jurisdicción especial, constituída por las autoridades de las comunidades campesinas y nativas. Por otro lado, el artículo $139^{\circ}$ del mismo cuerpo de leyes, señala, que no existe ni puede establecerse jurisdicción alguna independiente con excepción de la arbitral y militar.

53 Trazegnies, 1977, p. 98. 
Lo expuesto anteriormente, crea una duda acerca de la naturaleza de las potestades "jurisdiccionales" de las comunidades nativas y campesinas; pues para oscurecer aún más el panorama, la Constitución prevé además que la ley establecerá las formas de coordinación entre esta jurisdicción especial y los Juzgados de Paz y demás instancias del Poder Judicial.

Un análisis superficial de la normatividad citada, podría llevarnos a pensar que no se trata de una jurisdicción independiente, sino de una delegación de facultades del Poder Judicial para proveer de servicios legales a estas organizaciones.

Según la interpretación del texto por parte de Yrigoyen, se trataría de un defecto de sistemática, pues para ella es claro que esta jurisdicción especial es también independiente ${ }^{54}$. En opinión de Bustamante ${ }^{55}$, no debe entenderse que exista contradicción entre los citados artículos, pues el artículo $149^{\circ}$, no crea o establece una jurisdicción independiente, sino "reconoce vías alternativas que ya vienen funcionando exitosamente y les confiere vigencia constitucional"56.

En nuestra opinión, sí existe falta de sistemática al tratar la jurisdicción especial de la que hablamos, pues al conferirle la atribución de resolver conflictos con arreglo a su propio derecho (consuetudinario), y no existiendo límite en cuanto a materia, o sujetos, estamos hablando de una "jurisdicción", en el sentido técnico del término; más aún cuando éste no se encuentra subordinado al Poder Judicial, pues la ley habla solo de coordinación con sus diversas instancias.

\subsection{1 Ámbito de aplicación}

Las limitaciones impuestas en el ámbito territorial, resultan claras del texto de la norma, se trata del "territorio" de las comunidades.

Respecto de la elección de quienes deben ejercer estas potestades, se habla de las autoridades que, nosotros entendemos, son las que elija la propia comunidad sin posibilidad de intervención del Poder Judicial.

Yrigoyen ${ }^{57}$, en una posición que nosotros compartimos, señala que no se trata de una jurisdicción obligatoria, ya que existe libertad para

54 Yrigoyen, 1995, p.25 y 29.

55 Bustmante, 1993, p. 90.

56 Ibid.

57 Yrigoven, 1995, p. 41. 
las comunidades para determinar en qué casos desea intervenir, y si quiere intervenir.

Otro aspecto que suscita dudas se refiere a los sujetos, Yrigoyen se plantea una serie de posibilidades respecto de los comuneros que deseen ser juzgados por esta jurisdicción especial, aun cuando se trata de hechos o situaciones producidos fuera del espacio territorial de la comunidad $^{58}$. Al respecto se plantean dos respuestas con las que nosotros concordamos. Primero, si las partes en disputa son miembros de la comunidad, y los efectos de la decisión no implican a terceros ajenos a ella, es factible su uso en ejercicio del derecho a su identidad étnica y cultural ${ }^{59}$. Segundo, si una de las partes en conflicto es un tercero ajeno a ella pero acepta su competencia, el caso puede ser ventilado ante ésta ${ }^{60}$. Respecto de esta última opinión, nosotros deseamos precisar, que la elección del medio de solución es idéntica a si se tratase de cualquier otro medio (arbitraje, conciliación; etc.), por ende el individuo ajeno a ésta es libre de decidir la opción más beneficiosa para él, observando los límites que imponga un posible jurisdicción obligatoria del Poder Judicial respecto al caso que se discuta.

\subsubsection{Los derechos fundamentales}

La norma que comentamos señala que el derecho consuetudinario puede ser aplicado, siempre que no viole los derechos fundamentales de la persona.

Este es sin duda alguna, uno de los temas más difíciles de precisar, ya que nos encontramos en un campo abierto a la discusión, donde existen posiciones que niegan la universalidad de estos derechos, hasta aquellas que la consagran como derechos connaturales al hombre ${ }^{61}$.

Nosotros creemos que se trata de los supuestos mínimos y necesarios para la organización de una sociedad y su asimilación por todos los grupos humanos resulta indispensable para alcanzar niveles de vida dignos en cualquier sociedad o grupo de que se trate. No obstante ello,

58 Ibid., p. 42.

59 Ibid.

60 Ibid.

61 Puede consultarse el artículo de Eduardo Hernando Nieto, "¿Existen los derechos naturales", En: Derecho PUC, No 49, 1995. pp. 321-343. 
debemos reconocer que el contenido de estos no es siempre claro ni unívoco, por lo cual nos parece coherente el planteamiento de Yrigoyen, respecto de la posibilidad de hacer una "lectura pluricultural o multicultural"62 de ellos. Sin embargo, creemos que cualquier interpretación que se realice, debe por lo menos respetar el contenido mínimo que permita reconocer a estos derechos como tales.

Aunque suelen citarse algunos casos, en los cuales se llegó a disponer de la vida de algunos individuos juzgados ${ }^{63}$, Brandt sostiene que estos casos son excepcionales, y que las penas que imponen castigos corporales prácticamente pertenecen a la historia" ${ }^{64}$.

\subsection{El caso de Calahuyo}

En un estudio realizado en la Comunidad de Calahuyo (Puno), Peña Jumpa (1991) describe el funcionamiento del sistema de resolución de conflictos de esta comunidad.

Aunque el estudio comprende una descripción de los órganos y mecanismos de resolución, nosotros nos centraremos en un análisis de la correspondencia de esta evidencia empírica con respecto al postulado de eficiencia reclamado en el primer capítulo.

De acuerdo a lo señalado por Peña ${ }^{65}$, el origen del desarrollo de este sistema paralelo al de la justicia formal, se halla en un rechazo de la última a partir de la década del '70. Las razones que empujaron al rechazo de la justicia formal se encontrarían en lo ineficiente que resulta este sistema.

Algunas declaraciones de un comunero sistematizan las razones de este rechazo a "los jueces de la ciudad" ${ }^{66}$ :

"... una, debido a que "dichos señores cobran muy caro", dos, "porque les gusta alargar los juicios"; y tres en razón a que "al final no resuelven nada" ${ }^{67}$ (entrecomillado original).

62 Yrigoyen, 1995, p. 44.

63 Brandt, 1987, pp. 155-156, y 192.

64 Ibid. p. 150.

65 Peña, 1991, p. 271.

66 Ibid. p. 272.

67 rbid. 
Como podemos apreciar, la causa fundamental de esta elección por un mecanismo alternativo, obedece a lo oneroso que resulta el aparato formal, por lo cual es evidente que la decisión es "racional". En este caso, se suele mencionar tanto los costos originados por gastos "lícitos" (letrados, tasas), y los costes originados por la demora y las "coimas" que se ven obligados a pagar. En contraste, en la comunidad encontramos una justicia "barata" (en el mejor sentido del término) porque no hay que pagar nada, y además resulta más expeditiva.

La tercera razón alegada también resulta muy interesante, pues revelaría que en ese contexto el Poder Judicial no estaría cumpliendo su objetivo de acrecentar el bienestar social. En cambio, si tenemos una "justicia informal" que tiende a reducir el malestar social, pues del estudio se deduce que las decisiones son asumidas como "justas" por los interesados. La razón de esta efectividad, estribaría a nuestro entender en la "especialización" de las personas involucradas en la resolución de los conflictos (que les suelen ser muy familiares), lo cual reduciría grandemente la posibilidad de originar costos por errores judiciales, que se estaría manifestando en la frase "al final no resuelven nada".

La evidencia recogida por Brandt (1987), así como la afirmación realizada por Bustamante respecto a las comunidades cusqueñas ${ }^{68}$, nos permiten suponer que el caso de Calahuyo no es aislado sino que constituye la muestra de una eficiencia generalizada.

Un elemento interesante a ser analizado, es la presencia de instituciones informales, vinculadas al "respeto de la palabra empeñada" (sic), el cuidado del honor, o la reciprocidad como condiciones de la existencia de estos sistemas ${ }^{69}$. Es claro, como señala Peña ${ }^{70}$, que estas condiciones se dan porque las relaciones tienen un alto grado de personalización. Sin embargo cabe preguntarse, si este comportamiento claramente maximizador, se haría presente si las transacciones realizadas en las comunidades campesinas tuvieran una mayor relevancia en términos monetarios, o fueran menos personales. Nosotros creemos que la respuesta es afirmativa, pues los comuneros han demostrado un comportamiento claramente racional, limitado únicamente por las condiciones de extrema pobreza en que se encuentran, que los obliga

68 Bustamante, 1993 , p. 89.

69 Peña, 1991, p. 297.

70 Ibid. 
en algunas oportunidades a comportarse como minimizadores de ries$\operatorname{gos}^{71}$.

\section{A modo de conclusión}

Una primera sugerencia que podemos hacer, a la luz de lo expuesto en este trabajo, es la necesidad de que las reformas del sistema de administración de justicia consideren que el objetivo final no es reforzar al Poder Judicial sino acrecentar el bienestar social, por lo que es pertinente considerar la posibilidad de diversificar la "oferta de tutela de los derechos", mediante la promoción de mecanismos alternativos de solución de conflictos, como una alternativa de menor costo.

En segundo término, deseamos manifestarnos sobre la eficiencia subyacente en la decisión de las comunidades campesinas para resolver sus conflictos de una manera más barata y segura, aportando una solución no solo a sus problemas particulares sino al bienestar de la sociedad toda, pues además contribuyen al "descongestionamiento" de nuestro Poder Judicial. Nosotros estamos convencidos de que esta elección eficiente no es casual, sino que obedece al ánimo presente en los campesinos de la sierra de afrontar sus problemas de pobreza mediante la generación de más riqueza; y en este sentido, consideramos, que el patrón de comportamiento minimizador de riesgos, que citábamos al finalizar el segundo capítulo obedece únicamente al estado de pobreza extrema en que se hallan, pues para ellos una pequeña pérdida de sus ingresos puede resultar catastrófica. Creemos que urge una incorporación de estos sectores al mercado, lo cual estamos seguros, no importa la pérdida de sus patrones culturales.

\section{Referencias bibliográficas}

BeLAunde L. de R., Javier de

1991 "Aproximación a la realidad de la administración de Justicia en el Perú. En: Poder Judicial y democracia. García-

71 Figueroa, 1989, p. 92. 
Sayán (ed.). Lima: Comisión Andina de Juristas - Centro para la Independencia de Abogados y Jueces.

BRANDT, Hans-Jürgen

1987 Justicia popular: campesinos y nativos. Lima: Fundación Friedrich Naumann - CDIJ.

Bustamante B., Alberto

1992 Justicia alternativa. Lima: Instituto de Economía de Libre Mercado.

Cal.ABresi, Guido

1992 (1984) "Seguro de primera persona, de Tercera Persona y Responsabilidad por Productos ¿Puede el análisis económico del derecho decirnos algo al respecto?". En: Ius et Veritas, $N^{\circ} 4,1992$.

Calabresi, Guido y Melamed, Douglas

1992 (1972) "Reglas de propiedad, reglas de Responsabilidad e Inalienabilidad: Un Vistazo a la Catedral". En: Themis, $\mathrm{N}^{\circ} 21,1992$.

COASE, Ronald

1960 "The problem of the social cost". Journal of Law and economics, volumen III. pp. 1-44. (versión castellana trabajada sin referencia).

FigueroA, Adolfo

1988 Economía en la Sierra del Perú. (4a . Edición). Lima: Pontificia Universidad Católica del Perú.

Flores NANO, Lourdes

1995 Competencia Notarial en asuntos no contenciosos. En: Derecho Puc, $N^{\circ} 49$.

Friedman, Milton y Rose

1983 Libertad de Elegir. Barcelona: Ediciones Orbis S.A. 
García Sayán, Diego

1987 "Jueces letrados: el cuento de la vaca y la justicia". En: La Justicia de Paz y el Pueblo. Lima: CDIJ - Friedrich Naumann. pp. 39-43.

MiLl, John Stuart

1980 (1859) Sobre la libertad. Madrid: Aguilar.

North, Douglas C.

1981 Estructura y Cambio en la Historia Económica. Madrid: Alianza Editorial.

1990 Institutions, Institutional change and economic performance. Cambridge: Cambridge University Press.

PásARA, Luis

1982 Jueces, justicia y poder en el Perú. Lima: CEDYS.

Pastor Prieto, Santos

1984 "Una introducción al análisis económico del derecho". En: Hacienda Pública Española. Nº 89.

1993 ¡Ah de la Justicia! Política judicial y economía. Madrid: Civitas.

Peña Jumpa, Antonio

1991 "Justicia Comunal en Calahuyo". En: Allpanchis. N 37. pp. 269-303.

POLINSKY, A. Michell

1983 Introducción al Análisis Económico del Derecho. Barcelona: Ariel.

RAWLS, John

1985 Teoría de la Justicia. (2a. Edición). México: Fondo de Cultura Económica.

TORRES López, Juan

1987 Análisis Económico del Derecho. Madrid: Tecnos. 
Trazegnies, Fernando de

1977 "El caso Huayanay: el Derecho en situación-límite". En: Cuadernos Agrarios. No 1. pp. 73-118.

1996 Reflexiones sobre la Sociedad Civil y el Poder Judicial. Lima: ARA.

YrigoYen, Raquel

1995 Constitución, indígenas y derecho consuetudinario. Lima: CEAS - Desfaciendo Entuertos. 